\title{
What Happens after the COVID-19 Crisis: Managing "Patient Surge" in Academic Breast Imaging Division
}

\author{
Afnan Fahd Almuhanna*, \\ Department of Radiology, KFHU, Imam Abdulrahman Bin Faisal University, Dammam 31473, Saudi Arabia
}

\section{ARTICLE INFO}

\section{Article History}

Received 04 July 2020

Accepted 08 August 2020

Keywords

Covid-19

screening mammography

patient surge

abbreviated breast MRI

breast imaging preparedness

\begin{abstract}
The first case of coronavirus disease 2019 (COVID-19) in the Kingdom of Saudi Arabia was reported in the Eastern Province (Qatif City) on March 2, 2020 (Saudi Arabia Ministry of Health, https://www.moh.gov.sa/Pages/Default.aspx). Since then, a cluster of cases continued to expand and spread throughout the kingdom. Several procedures were implemented to contain the pandemic. By May 8, 2020, the Saudi Center for Disease Prevention and Control had reported a total of 35,432 cases (https:// covid19.cdc.gov.sa/daily-updates/). As of this writing, the projected peak of cases in Saudi Arabia occurred from April 24 to May 10, 2020. "Bending of the curve" will extend this curve to June and July, with a theoretically ending date of September 10, 2020 (Predictive Monitoring of COVID-19 developed by SUTD Data-Driven Innovation Lab, https://ddi.sutd.edu.sg). However, social distancing practices will remain in force until mid-2021 (Ferguson et al., Imperial College London, https://spiral.imperial. ac.uk:8443/handle/10044/1/77482). This pandemic affected every aspect of people's lives with a significant impact on medical education and healthcare services, including breast imaging. In this article, the author lists some of the possible solutions for problems relating to patient surge once stay-at-home orders are relaxed.
\end{abstract}

(C) 2020 Dr. Sulaiman Al Habib Medical Group. Publishing services by Atlantis Press International B.V. This is an open access article distributed under the CC BY-NC 4.0 license (http://creativecommons.org/licenses/by-nc/4.0/).

\section{BREAST CANCER SCREENING IN SAUDI ARABIA}

Breast cancer is the most common type of cancer among Saudi women according to the Saudi Cancer Registry Report released in 2015 [1], The first breast cancer screening center was established in Riyadh, Saudi Arabia, via a nongovernmental collaboration between Abdul Lateef Charitable Center and the Saudi Cancer Society in 2007. Another nongovernmental screening program was initiated in the Eastern Province using mobile mammogram machines through a collaboration between Imam Abdulrahman Bin Faisal University (formerly known as Dammam University) and the Saudi Cancer Foundation in 2009. The national screening intuitive was launched by the Ministry of Health in 2015-2016. However, the process of seeking screening for breast cancer is not clear, and no available data in this regard can be found at their official website.

Since the start of our screening program in 2009 at our university hospital $[2,3]$, we committed ourselves to continue operation despite all obstacles. However, with the crisis brought by the COVID-19 pandemic, most of the breast imaging practices were scaled down or went into temporary shutdown in our region.

Our primary aim is to ensure the safety of our patients as well as staff in addition to preserving Personal Protective Equipment (PPE) to cope with hospital shortage; as a result, we need to prioritize our cases accordingly.

"Email:amuhanna@iau.edu.sa

Peer review under responsibility of the Dr. Sulaiman Al Habib Medical Group

Data availability statement: Data available upon request.
This pandemic brought two challenges: maintaining operation during the pandemic and planning for patient surge in the near future. For this reason, three phase reoperation plans were designed that place importance on Continuous Performance tracking, monitoring, and adaptation as needed during these three phases.

\section{GENERAL RECOMMENDATIONS TO ENSURE PATIENT AND STAFF SAFETY}

- Implementation of social distancing procedures and adequate gap between imaging appointments to enhanced room and equipment cleaning, 10-15-min interval between patients in mammography, breast ultrasound, and breast Magnetic Resonance Imaging (MRI), and 15-min interval between interventional procedures.

- Extension of operation hours, including evenings and weekends, to allow for adequate spacing.

- All breast imaging studies should be delayed in cases of known or suspected COVID-19 patients.

- Phone call scheduling to limit potential patient exposures, screening patients for fever and symptoms of COVID-19 upon entry, masking all patients as well as all hospital employee.

- Ensure adequate supply of hand sanitizers in all work areas and patient contact areas, including front desks, with emphasis on importance of hand hygiene practices. 
- Ensure adequate supply of PPE.

- Continuance of remote consultation via smart calls or video calls, virtual multidisciplinary meetings and educational conferences.

- Continuance of social distancing practices in reading rooms, waiting areas, and radiology front desks.

- Continuance of online teaching for under- and postgraduate students through zoom video communication.

- Enhancement of research capability and providing opportunities for students to participate.

\section{REOPERATION PHASES}

All phases were designed in multidisciplinary meetings with the breast care team including surgeons, oncologists, and pathologists.

\subsection{Phase 1 (Diagnostic Reoperation Phase for Urgent Cases)}

- Enhancement of walk-in clinics. Most diagnostic studies for clinically suspicious patients, including mammography and ultrasound, should be performed on the same day at the clinic every Thursday; thus, all scheduled nonurgent cases will have to be cancelled.

- Urgent follow-up appointments for clinically suspicious cases.

- Urgent appointments for Breast Imaging-Reporting and Data System (BI-RADS) 4 (suspicious) and BI-RADS 5 (highly suggestive of malignancy) biopsies [4].

- Assessment of treatment response should be done earlier at the same day of planned next chemotherapy cycle.

- Resumption of BI-RADS 3 (probably benign) patient appointments [4].

- Diagnostic MRI should be limited for patients with pathologyproven malignancy to assess the extension of disease in particular to Type D (extremely dense) and Type C breast (heterogeneous dense) parenchyma.

- Revise MRI protocols and try to utilize limited sequences to limit patient scan time and enhance MRI workflow.

\subsection{Phase 2 (High-risk Screening Reoperation Phase for Semiurgent Cases)}

- Limit our cases to patients with high-intermediate risk (15-20\% lifetime risk), with a suggestion to relay only on tomosynthesis images and reconstructed mammography to reduce examination time.
- Resume routine follow-up for treated cancer patients.

- Radiologists should be around during examination time to lower BI-RADS 0 (Incomplete), thereby reducing recall rate [4].

- For screening MRI, use abbreviated protocol (10-min examination) or Ultrafast breast MRI (5-min examination) in conjunction with mammography to reduce examination time $[5,6]$.

\subsection{Phase 3 (Screening Reoperation Phase for Nonurgent Cases)}

Back to routine screening for low-risk patients but under strict regulations provided by the infection control department and hospital administration listed in general recommendations.

\section{CONCLUSION}

Within the next few months, hospitals will need to continue to act aggressively in their responses to address the potentially ongoing spread of COVID-19, and compensate for lost appointments including imaging. Breast imagers should be ready for reoperation in the near future, a routine that will never be like past normal.

\section{CONFLICTS OF INTEREST}

The author declares that there are no conflicts of interest regarding the publication of this paper.

\section{REFERENCES}

[1] Saudi Cancer Registry Report. Available from: https://nhic.gov. sa/eServices/Documents/E\%20SCR\%20final\%206\%20NOV.pdf (accessed May 9, 2020).

[2] Al Mulhim FA, Syed A, Bagatadah WA, Al Muhanna AF. Breast cancer screening programme: experience from Eastern province, Saudi Arabia. East Mediterr Health J 2015;21;111-19.

[3] Abulkhair OA, Al Tahan FM, Young SE, Musaad SMA, Jazieh ARM. The first national public breast cancer screening program in Saudi Arabia. Ann Saudi Med 2010;30;350-7.

[4] D’Orsi CJ, Sickles EA, Mendelson EB, Morris EA. ACR BI-RADS atlas: breast imaging reporting and data system. Reston, VA: American College of Radiology; 2013.

[5] Chhor CM, Mercado CL. Abbreviated MRI protocols: wave of the future for breast cancer screening. AJR Am J Roentgenol 2017;208;284-9.

[6] Mann RM, Cho N, Moy L. Breast MRI: state of the art. Radiology 2019;292;520-36. 